\title{
Globe
}

Revue internationale d'études québécoises

\section{Pierre Serré : Deux poids, deux mesures. L'impact du vote des non-francophones au Québec. Montréal, VLB éditeur, 2002}

\section{Jean Crête}

Volume 7, numéro 1, 2004

URI : https://id.erudit.org/iderudit/1000839ar

DOI : https://doi.org/10.7202/1000839ar

Aller au sommaire du numéro

Éditeur(s)

Globe, Revue internationale d'études québécoises

ISSN

1481-5869 (imprimé)

1923-8231 (numérique)

Découvrir la revue

Citer ce compte rendu

Crête, J. (2004). Compte rendu de [Pierre Serré : Deux poids, deux mesures.

L'impact du vote des non-francophones au Québec. Montréal, VLB éditeur, 2002].

Globe, 7(1), 214-217. https://doi.org/10.7202/1000839ar d'utilisation que vous pouvez consulter en ligne.

https://apropos.erudit.org/fr/usagers/politique-dutilisation/ 
Contrairement à Cixous qui prône l'inscription de la différence sexuelle à travers l'élaboration d'une "écriture féminine", "Hyvrard prefers to avoid [thisl category because it could too easily become another way of excluding women from the recognition of the meaning of their literary quests to speak the unspoken, to express what has beretofore remained silent, and to find a way to speak of difference which is not oppositional." (p. 266)

En somme, l'ouvrage de Santoro se veut un panorama transatlantique d'une littérature à la fois féministe et d'avant-garde. L'auteure prend soin de distinguer ces deux termes et précise que l'écriture féministe des années 1970, si elle présente des points communs avec l'avant-garde, s'en éloigne par sa portée éthique : " The effect of the literary innovations and etbical commitment of avant-garde feminist texts bas no doubt been amplified and sustained in part by the rise of feminist criticism and its institutionalization in programs dedicated to women's studies " (p. 279). La principale force de Mothers of Invention réside sans doute dans la richesse de l'éventail textuel proposé, l'auteure puisant abondamment à même les autres productions des écrivaines étudiées. Si Santoro en arrive à des conclusions analogues à celles de la critique féministe au sujet de l'avant-garde littéraire, il n'en demeure pas moins qu'elle parvient à mettre en lumière la spécificité des textes retenus. L'analyse proposée est donc à l'image des romans présentés : ouverte et axée sur la différence dans le même.

Lucie Guillemette et Marie-Claude L'Heureux Université du Québec à Trois-Rivières

\section{Pierre Serré}

Deux poids, deux mesures.

L'impact du vote des non-francopbones au Québec. Montréal, VLB éditeur, 2002.

Dans cet ouvrage, Pierre Serré étudie les conséquences du mode de scrutin majoritaire lorsqu'un groupe majoritaire électoralement divisé fait face à un groupe minoritaire unifié. La monographie porte sur le Québec et le groupe minoritaire dont il traite est bien sûr constitué des électeurs 
non francophones. La thèse de l'auteur est que, dans certains cas de figure, le mode de scrutin majoritaire introduit des distorsions et des effets perturbateurs dans la représentation à l'Assemblée nationale, mais aussi plus particulièrement dans la composition du Conseil des ministres.

Après avoir fait le point sur les recherches antérieures, Serré propose trois façons de mesurer la structure de l'électorat afin d'évaluer ensuite le degré de dépendance d'un parti à l'égard d'un groupe minoritaire. Quel est le poids numérique d'un tel groupe par rapport à l'ensemble de la clientèle d'un parti ? Quel est le degré de volatilité du comportement du groupe minoritaire? Comment les résultats électoraux se traduisentils en sièges?

Les données sur lesquelles portent l'analyse sont nombreuses et complexes, mais systématiques, et proviennent des élections générales et des référendums tenus de 1970 à 1998. Les unités observées sont bien sûr les circonscriptions électorales auxquelles s'ajoutent, pour la région métropolitaine de Montréal, les secteurs de recensement, parfois regroupés en quartiers (un secteur de recensement est une unité géographique qui compte en moyenne 4000 personnes). On dénombre 550 de ces unités en 1970, alors qu'il y en a 755 en 1998 . Tous les analystes qui ont eu à transcrire les données électorales dans des unités de recensement apprécieront la patience et la minutie de l'auteur. Heureusement pour le lecteur, les informations sont présentées de façon très claire dans le texte, mais aussi à l'aide de tableaux et de cartes.

Une des variables clefs de l'étude est la langue. L'auteur reprend la division classique au Canada de trois groupes linguistiques, définis selon la langue parlée à la maison : les anglophones, les francophones et les allophones. L'utilisation de cette dernière catégorie, qui fut introduite il y a quelques années pour fins de construction sociale, et non d'analyse scientifique, nous semble malheureuse en ce qu'elle introduit un certain vague. En clair, le fait de ne parler ni français ni anglais à la maison est un indicateur indirect et incomplet de ce que l'on veut mesurer et est partiellement chargé d'informations non pertinentes qui encombrent l'analyse. L'auteur le concède d'ailleurs de façon implicite dans une note (note 14) de la conclusion de l'ouvrage.

Parce que les nouveaux citoyens et les anglophones sont surtout présents dans l'agglomération montréalaise, cette dernière est scrutée à la loupe dans chacun des secteurs de recensement. L'auteur y compare 
l'inscription sur les listes électorales, l'abstention lors des scrutins et la mobilisation, une mesure combinant inscription et abstention, pour montrer que les francophones ont finalement plus de poids dans l'électorat votant que dans la population. Le Parti québécois $(\mathrm{PQ})$ recevrait presque tous ses votes de francophones alors que le Parti libéral du Québec (PLQ), stable chez les anglophones, gagnerait peu à peu le vote des allophones en même temps qu'il perdrait des votes francophones. Par ailleurs, la comparaison de toutes les régions du Québec souligne à nouveau que c'est le centre-ville montréalais qui était et qui demeure le plus favorable au PQ alors qu'en dehors de la région de Montréal, ce sont les milieux ruraux ou faiblement urbanisés qui ont toujours le plus fortement appuyés le PLQ. En utilisant la distribution des électeurs selon les caractéristiques linguistiques il est possible, selon Serré, de prédire avec assez d'exactitude l'élection ou non d'un candidat libéral ou péquiste.

L'auteur s'intéresse au premier chef à décrire l'impact du vote unifié d'une minorité sociale sur la vie politique et résume ainsi la situation: "La proportion de non-francophones dans la population totale tourne autour des $18 \%$, leur proportion dans l'ensemble des votants est d'environ $15 \%$, leur part dans l'ensemble de la clientèle libérale se situe en moyenne à $30 \%$ tandis que la proportion des sièges marqués par l'impact du vote des non-francophones sur le total des sièges libéraux s'élève à $73 \%$. " (p. 176). Comment expliquer cette influence disproportionnée de la minorité linguistique?

Lorsque le PLQ est dans l'opposition, ces sièges sont surtout assurés par les circonscriptions où la minorité linguistique est importante. Lorsque le PLQ prend le pouvoir, les députés élus dans ces circonscriptions ont plus de chance que les autres de devenir ministres et même de détenir les portefeuilles les plus importants. Cela pourrait s'expliquer par une plus grande expérience parlementaire, quoique ces personnes sont souvent parachutées dans des circonscriptions sûres justement parce qu'on souhaite s'assurer de leur accession au Conseil des ministres. Pour corriger ces effets pervers et d'autres distorsions, l'auteur propose de modifier le mode de scrutin pour le rendre proportionnel. La proportionnalité, selon lui, permettrait d'apaiser le climat tendu qui règne entre anglophones et francophones et favoriserait l'apparition de meilleures conditions pour débattre d'enjeux de société. 
L'étude de Pierre Serré est solide et conduite logiquement. Ses résultats, pris un à un, sont validés par de nombreuses autres études partielles ou qui ont traité par le biais les mêmes questions. Le grand apport de Serré est de montrer de façon systématique l'enchaînement logique à la source d'une réalité déjà connue et bien observée. Un groupe minoritaire unifié, ici les anglophones et les nouveaux citoyens qui s'associent aux anglophones, réussit à utiliser ses ressources électorales avec grand succès, ce qui semble a priori fort souhaitable si ce n'était du fait que l'électeur médian n'est pas très bien servi par cette dynamique. L'étude de Serré a aussi le mérite de lier la sociologie électorale et l'étude des institutions en ces années où l'on s'interroge sur la réforme des institutions électorales au Québec. La publication de cet ouvrage ne pouvait pas survenir à un meilleur moment.

Jean Crête

Université Laval

\section{Yannick Gasquy-Resch}

Gaston Miron, le forcené magnifique.

Montréal, HMH, coll. "América ", 2003.

Les passions, surtout naissantes, se nourrissent de toute ligne concernant leur objet. C'est notamment ce qui confère aux introductions de nature biographique une importance non négligeable. Quelques exemplaires de la collection "Écrivains de toujours " conservent, dans tels plis de ma bibliothèque, le souvenir de lectures voraces passées à entretenir des enthousiasmes verts, issus quelquefois de détails insignifiants mais d'une portée personnelle insoupçonnée. Ces livres apparaissent ainsi comme de précieux relais de contagion.

C'est à la catégorie des ouvrages introductifs qu'appartient Gaston Miron, le forcené magnifique, un "essai biographique " destiné à un public non spécialiste, mais qui aime la poésie . Quoique publié au Québec, le livre participe manifestement du désir de diffuser une œuvre "hors des frontières qui l'ont vule] naître " (p. 17) et paraît, en ce sens, se destiner essentiellement au lecteur non québécois - en témoigne le "petit glossaire mironien * et ses entrées telles que "maganer ", "giguer ", 